\title{
Errors in Tropical Cyclone Intensity Forecast by RSMC Tokyo and Statistical Correction Using Environmental Parameters
}

\author{
Kosuke Ito \\ University of the Ryukyus, Okinawa, Japan
}

\begin{abstract}
Tropical cyclone (TC) intensity forecasts issued by the Regional Specialized Meteorological Centre (RSMC) Tokyo - Typhoon Center are systematically compiled to analyze the long-term behavior of errors and to explore the potential for improvement in the forecast accuracy using a statistical correction approach. In this study, a dataset is constructed from annual statistics and every single forecast listed on annual reports on the activities of the RSMC Tokyo. This study found that (1) the accuracy of annual mean forecast has not improved over 26 years and that (2) forecast errors tend to be larger in the rapidly developing TCs. Further analysis reveals that recent forecast output (2008-2014) contains biases associated with the magnitude of the vertical shear of horizontal wind, convective available potential energy, upper ocean temperature, maximum potential intensity (MPI) and ocean coupling potential intensity (OC_PI). To evaluate the adverse effect of such biases in the current forecast system, a simple statistical correction is applied. It improved TC intensity forecast by $7.8-16.9 \%$ when an OC_PI is employed.
\end{abstract}

(Citation: Ito, K., 2016: Errors in tropical cyclone intensity forecast by RSMC Tokyo and statistical correction using environmental parameters. SOLA, 12, 247-252, doi:10.2151/sola.2016049.)

\section{Introduction}

The forecasts of tropical cyclone (TC) intensity have been of particular importance in the atmospheric sciences because of the potential severity of resulting disasters. Since 1 July 1989 , the Japan Meteorological Agency (JMA) has been serving as a Regional Specialized Meteorological Centre (RSMC) Tokyo - Typhoon Center issuing TC advisories in the Western North Pacific (WNP) within the framework of the World Weather Watch program of the World Meteorological Organization. As the WNP contains the largest number of very intense TCs of any region (D'Asaro et al. 2011), official forecasts published by the RSMC Tokyo benefit many circum-Pacific countries. It is generally believed that forecasts of TC intensity, represented by minimum sea level pressure (MSLP) and 10-min averaged maximum wind speed at $10 \mathrm{~m}$ height (Vmax), has been challenging comparing to track forecasts (Wang and Wu 2004). For example, DeMaria et al. (2014) demonstrated that the best intensity guidance model exhibits the statistically significant improvements in the WNP over three decades, but not official intensity forecasts conducted by the Joint Typhoon Warning Center. Yu et al. (2013) analyzed the output of the JMA global spectral model (JMA-GSM), which is currently used for RSMC Tokyo's official TC intensity forecast, and has proven to be useful for intensity forecasts. However, errors in official intensity forecasts issued by the RSMC Tokyo have never been investigated in detail to the author's knowledge; these forecasts comprise a synthesis of the capability of numerical models, statistical corrections, and the forecaster's skills. Considering the social and economic importance of the RSMC Tokyo's official forecasts, it is crucial to investigate the nature of their intensity errors and, if possible, to assess improvement

Corresponding author: Kosuke Ito, University of the Ryukyus, 1 Sembaru, Nishihara 903-0213, Japan. E-mail: itokosk@sci.u-ryukyu.ac.jp. (C2016, the Meteorological Society of Japan. possibilities by relating the errors of TC intensity forecasts to the environmental conditions, as Bhatia and Nolan (2013) did for hurricanes in the Atlantic Ocean.

The first aim of this work is to construct and analyze a dataset of intensity (MSLP and Vmax) forecast errors defined as the deviation in RSMC Tokyo official forecast relative to the RSMC Tokyo best track. The second aim is to quantify biases (defined as a composite mean error in a category) in regard to environmental parameters. Finally, we evaluate the potential for improvement using environmental parameters to diminish biases through a statistical correction. They contribute to reviewing the behavior of TC intensity forecast errors issued by the RSMC Tokyo and to the design of future TC intensity forecast systems.

\section{Datasets}

Figure 1 briefly summarizes the history of TC intensity forecasts, available error data, and numerical models in JMA. To date, the JMA has been primarily employing a JMA-GSM with a grid spacing of $20 \mathrm{~km}$ as a base model since November 2007 and applying corrections to numerical model results based on a Dvorak CI number and MSLP relationship, sea surface temperature (SST) and MSLP relationship, and statistical forecasts in order to publish the official forecasts (Koide et al. 2014). Official sixhourly individual intensity forecast errors have been recorded in the appendices of RSMC Tokyo annual reports (hereafter referred to as ARs) since 1992 in addition to annual errors available since 1989. For Vmax errors, both knots and meters per second are used as the unit in ARs depending on the year; we converted knots to meters per second using $1 \mathrm{knot}=0.5144 \mathrm{~m} \mathrm{~s}^{-1}$. Forecast accuracy is not verified before a TC reaches the threshold of tropical storm status or an extratropical cyclone subjected to transition from the TC.

To relate the forecast errors and environmental physical parameters, we focus on the period from 2008 to 2014, following the implementation of the JMA-GSM in November 2007 because their relationship is dependent on a base numerical model in addition to guidance, and quality of the best track. Environmental physical parameters considered here are the magnitude of the vertical shear of horizontal wind, ambient convective available potential energy (CAPE), surface temperature, upper ocean temperature (UOT), and maximum potential intensity (MPI), which are relevant to the TC intensity (e.g. Emanuel et al. 2004; Lin et al. 2013; Wang and Wu 2004). MPI was originally proposed by Emanuel (1986) as a theoretical upper limit of MSLP and Vmax given ideal environmental conditions, including fixed SST, for an axisymmetric vortex. We employ the version of this concept revised by Bister and Emanuel (1998), hereafter referred to as E-MPI. We also tested an ocean coupling potential intensity (OC PI) proposed by Lin et al. (2013) as an index in which the SST of E-MPI is replaced with the depth-averaged UOT, to account for the atmosphere-ocean coupling effect.

The magnitude of the vertical shear is defined as the difference of wind vectors between $850 \mathrm{hPa}$ and $200 \mathrm{hPa}$, averaged within $300 \mathrm{~km}$ from the TC center, as described by Ueno and Kunii (2009). UOT is represented by the depth-averaged ocean temperature from surface to $100 \mathrm{~m}, T_{100}$ (Price 2009). E-MPI for Vmax and MSLP are expressed as follows (Bister and Emanuel 1998, 2002): 


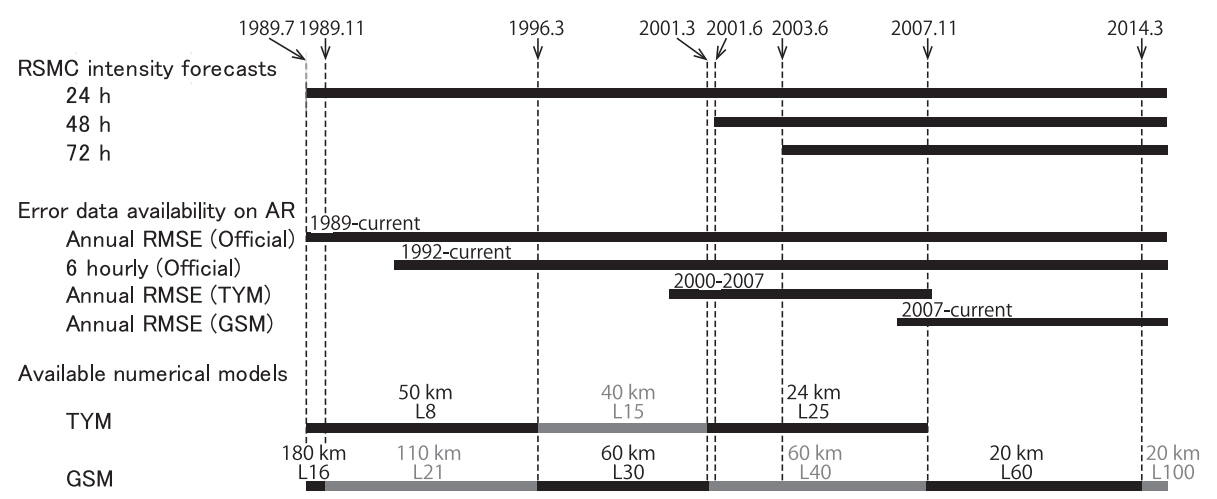

Fig. 1. History of TC intensity forecasts, error data availability, and numerical models in the JMA. The horizontal grid spacing (in km) and the number of vertical layers (beginning with L) are also shown for available numerical models. TYM represents a TYphoon Model, which was a model formerly used in JMA.

$$
\begin{aligned}
& V_{\max }^{2}=\frac{T_{S}-T_{O}}{T_{O}} \frac{C_{k}}{C_{D}} C_{p} T_{S}\left(\ln \theta_{e, S}^{*}-\ln \theta_{e, B}\right) \\
& 2 C_{p} T_{S} \ln \frac{P_{v}}{P_{c}}=V_{\max }^{2} \\
& 2 C_{p} T_{S} \ln \frac{P_{e n v}}{P_{v}}=V_{\max }^{2}+C A P E_{B}
\end{aligned}
$$

where $V_{\max }$ is the maximum wind speed, $P$ is the sea-level pressure, $C_{D}$ is the drag coefficient, $C_{k}$ is the surface enthalpy exchange coefficient (assuming $C_{D}=C_{k}$ as in Emanuel (1986)), $C_{p}$ is the specific heat at constant pressure, $T$ is temperature, $\theta_{e}{ }^{*}$ is the saturated equivalent potential temperature and $\theta_{e}$ is the equivalent potential temperature. $C A P E_{B}$ is the $C A P E$ of boundary layer air under the eyewall. Subscripts $S, O, B, c, v$ and $e n v$ represent the values at sea surface, outflow layer, boundary layer, TC center, the radius of Vmax, and environment, respectively. The actual algorithm is based on the work of Bister and Emanuel (2002). The outflow layer is defined at the level of neutral buoyancy. To calculate OC PI, $T_{S}$ in E-MPI is replaced with $T_{100}$. Note that OC PI cannot be calculated where UOT to a depth of $100 \mathrm{~m}$ is not available. For fair comparison between E-MPI and OC PI, E-MPI is referred to as E-MPIO when OC PI is available. E-MPIL refers to E-MPI applied to coastal regions or land, representing about $8 \%$ and $10 \%$ of total forecast cases, respectively.

Wind fields, atmospheric temperature, humidity and ground temperature data (including SST) are obtained from the operational version of JMA-GSM and Merged Satellite and In-situ Data Global Daily SST (See Sections 3.2 and 5.2 of JMA 2013). Six-hourly data is stored with a grid spacing of $0.5^{\circ}$ by $0.5^{\circ}$ in the Kyoto University Research Institute for Sustainable Humanosphere database (available at http://database.rish.kyoto-u.ac.jp/ arch/jmadata/data/gpv/original/). The daily UOT is retrieved from the Four-dimensional Variational Ocean ReAnalysis for the WNP over 30 years (Usui et al. 2016, manuscript submitted to $J$. Oceanogr.), based on the JMA Meteorological Research Institute Multivariate Ocean Variational Estimation system (Usui et al. 2006). Because a $4 \mathrm{D}$-Var-based fine-mesh dataset $\left(15^{\circ} \mathrm{N}-60^{\circ} \mathrm{N}\right.$ and $118^{\circ} \mathrm{E}-180^{\circ} \mathrm{E}$ ) with a horizontal grid spacing of $0.1^{\circ}$ does not cover the whole region which the RSMC Tokyo is responsible for, we used the 3D-Var-based parent domain output with the grid spacing of $0.5^{\circ}$.

During analysis, the vertical shear is centered at the location of MSLP, based on the JMA-GSM rather than the best track, and is temporally averaged over the integration time. Environmental CAPE, ground temperature (including SST), UOT, and the relative humidity are defined as the values at the TC center position in the best track, 2 days prior to the verification time (Lin et al. 2013). For simplicity, the temperature and humidity at the lowest atmospheric level of JMA-GSM are used for the values of boundary layer.

\section{TC intensity error}

Figure 2 shows annual mean forecast errors in TC position (including track forecasts before JMA started to serve as RSMC Tokyo), MSLP and Vmax. While track forecast errors today have decreased to less than half of those obtained 30 years ago, the long-term trend of intensity forecast error exhibits an increasing behavior. It might be because of the recent increase of rapid intensification $^{1}$ events rather than the degeneration of the forecast system as discussed in Section 5. Mean biases of MSLP and Vmax are closer to zero on average for forecast time (FT) of 24 and $48 \mathrm{~h}$, whereas forecasts of TC intensity have typically exceeded actual intensity at $\mathrm{FT}=72 \mathrm{~h}\left(-4.3 \mathrm{hPa}\right.$ and $+3.4 \mathrm{~m} \mathrm{~s}^{-1}$ on average $)$ in the last seven years (figures not shown).

We classify forecast errors based on the development rate defined as the Vmax change in the $24 \mathrm{~h}$ preceding a verification time (Fig. 3). Figure 3 a shows that MSLP forecasts generally have a positive bias for intensifying TCs and a negative bias for decaying TCs, suggesting that forecasts fail to capture the amplitude of intensity change in the rapid intensification and decay. These biases contribute to larger RMSEs in the forecast of intensifying and decaying TCs (Fig. 3b). These features are generally consistent with the quality of Vmax forecasts (data not shown).

Mean biases and standard deviations of MSLP in the forecasts of RSMC Tokyo are categorized according to environmental conditions for open ocean cases (Fig. 4). Corresponding figures for the coastal region and land are shown in supplemental material A. Figure $4 \mathrm{a}$ shows that a MSLP forecast tends to be much lower than the best-track MSLP, which might have caused false alarms, when the magnitude of vertical shear exceeds $8 \mathrm{~m} \mathrm{~s}^{-1}$. The vertical wind shear works to suppress TC intensity (Wang and Wu 2004), but weakening processes associated with vertical wind shear may not be sufficiently reproduced. The MSLP forecast expresses a negative bias when CAPE is lower than $1000 \mathrm{~J} \mathrm{~kg}^{-1}$ or $T_{S}$ is lower than $27^{\circ} \mathrm{C}$ (Figs. $4 \mathrm{~b}$ and c). Figure $4 \mathrm{~d}$ shows that MSLP forecast tends to be too low when $T_{100}$ is lower than $27^{\circ} \mathrm{C}$, and too high when $T_{100}$ is approximately $29^{\circ} \mathrm{C}$ or higher. Since TCs are more likely to intensify with a higher $T_{100}$, it may bring about failures to capture TC intensification. Biases mentioned above are statistically significant with at least the $95 \%$ confidence level. Figures $4 \mathrm{e}$ and $\mathrm{f}$ indicate that RSMC Tokyo predicts excessively weak TCs over the ocean when E-MPI or OC_PI expects very intense TCs and vice versa. It is notable that the coefficient of correlation between an error and the deviation for MSLP in OC_PI was -0.33 at $\mathrm{FT}=72 \mathrm{~h}$, while that between an error and the deviation in E-MPIO was -0.27 . This indicates a close relationship between

\footnotetext{
${ }^{1}$ Here, the rapid intensification (decay) is defined as an increase (decrease) in the 10-min maximum sustained winds of more than $15 \mathrm{~m} \mathrm{~s}^{-1}$ in a $24-\mathrm{h}$ period. It roughly corresponds to the definition employed in National Hurricane Center (NHC), while NHC uses a 1-min averaged maximum sustained winds.
} 
(a)

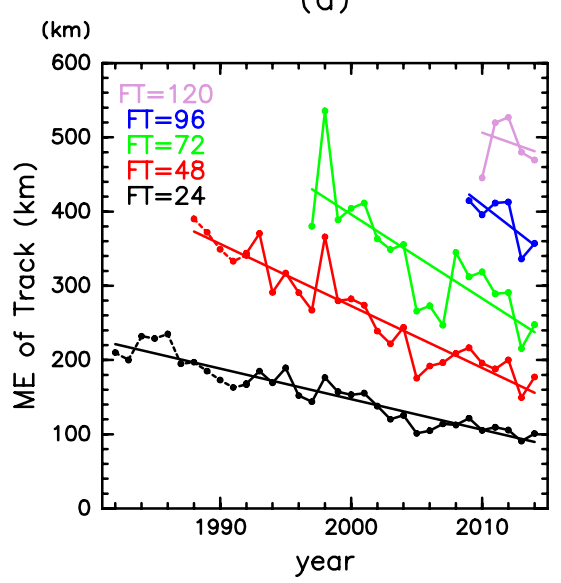

(b)

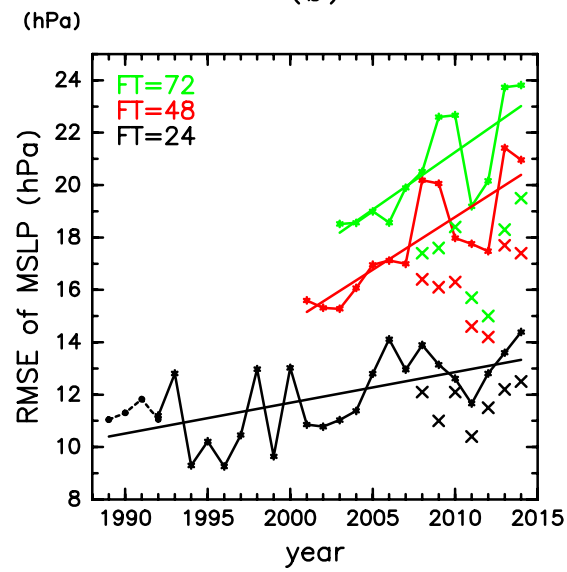

(c)

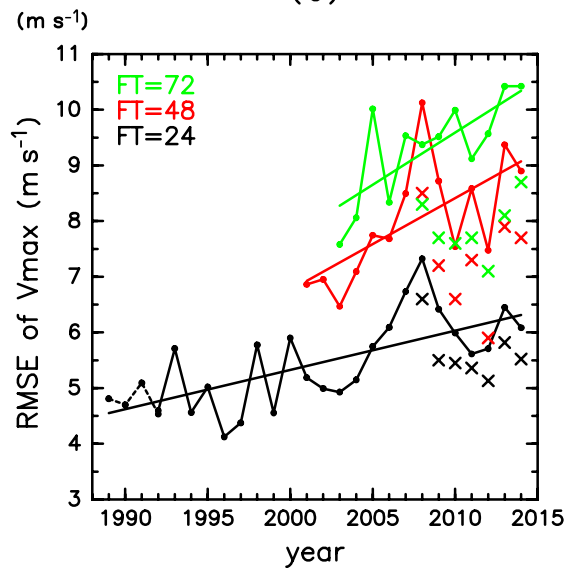

Fig. 2. Annual mean TC forecast errors in RSMC Tokyo, (a) track (b) MSLP (c) Vmax (with closed circles). Note that the annual statistics reported from 1989-1992 are plotted with dotted lines, while annual mean values from 1992-2014, which are computed by each forecast error, are plotted with solid lines. Regression lines are also drawn for annual mean values without closed circles. Cross marks represent RMSEs for statistically corrected forecasts of MSLP and Vmax in Section 4.

(a)

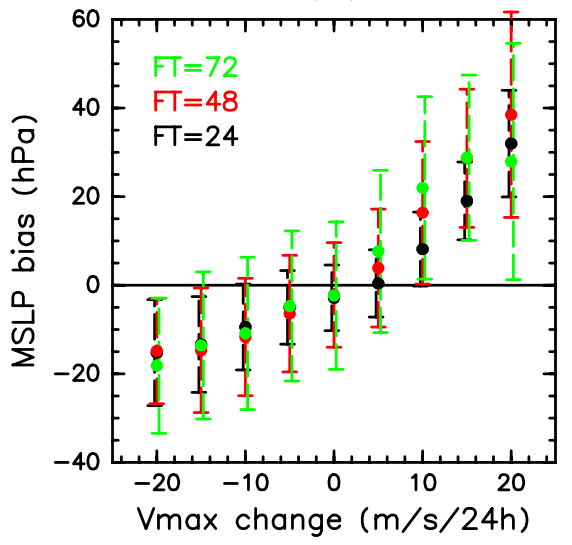

(b)

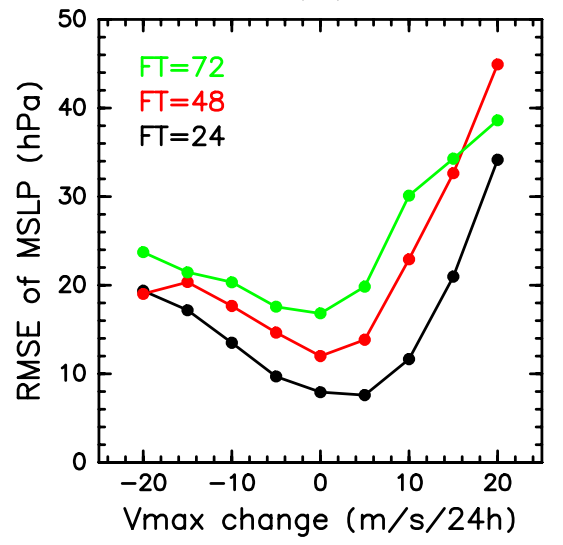

(c)

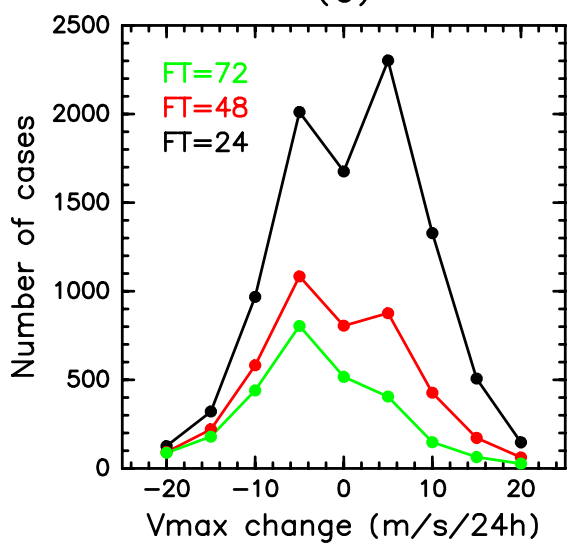

Fig. 3. (a) MSLP biases, (b) RMSE of MSLP, and (c) number of cases, categorized according to Vmax change in the last $24 \mathrm{~h}$ prior to time of verification.

intensity error and an index that accounts for atmosphere-ocean coupling. The above-mentioned biases generally become larger with increasing FT, highlighting the large benefits of the correction with environmental parameters in improving a relatively long-term forecast.

Biases in Vmax are consistent with those in MSLP (Fig. 5) ${ }^{2}$. That is, excessively intense TCs were predicted in RSMC Tokyo official forecasts when the vertical wind shear was greater than $8 \mathrm{~m} \mathrm{~s}^{-1}$, when CAPE was lower than $1000 \mathrm{~J} \mathrm{~kg}^{-1}$, when $T_{S}$ or $T_{100}$ was lower than $27^{\circ} \mathrm{C}$, or when potential intensities expected relatively weak TCs compared to RSMC Tokyo forecast. Inversely, excessively weak TCs were predicted when $T_{100}$ was approximately $29^{\circ} \mathrm{C}$ or higher, or when potential intensities expected very intense TCs.

\section{Statistical correction}

In the previous section, we found biases in official forecasts by stratifying the data according to several environmental parameters. Hence, information on environmental parameters is useful to diminish intensity errors with almost no additional computational

\footnotetext{
${ }^{2}$ MSLP was replaced by Vmax in Fig. 5. Therefore, the features of Figs. $5 \mathrm{a}, \mathrm{b}, \mathrm{c}$, and d generally have an opposite sign to those of Figs. 4a, b, c, and $d$ axis, while the features of Figs. $5 \mathrm{e}$ and $\mathrm{f}$ have a same sign to those of Figs. $4 \mathrm{e}$ and $\mathrm{f}$ axis.
}

cost.

As a first step to evaluate room for improvement, the following simple linear regression is considered:

$$
\begin{aligned}
F^{\prime}= & F+\alpha_{1} \text { SHEAR }+\alpha_{2} \mathrm{CAPE}+\alpha_{3} \mathrm{MSLP} \_ \text {PI } \\
& +\alpha_{4} \text { MSLP_RSMC }+\beta+\varepsilon
\end{aligned}
$$

where $F$ and $F^{\prime}$ represent the official TC intensity forecast issued by RSMC Tokyo and a statistically corrected forecast, respectively. The coefficients $\alpha_{1}, \alpha_{2}, \alpha_{3}, \alpha_{4}$ and $\beta$ are determined for each forecast time $(\mathrm{FT}=24,48,72 \mathrm{~h})$ and each TC intensity metric (MSLP or Vmax) using a linear regression analysis to minimize residual $\varepsilon$. MSLP PI represents potential intensity for MSLP either based on E-MPI or OC_PI. The dataset is divided into seven groups depending on the year (2008-2014). In order to correct the official forecasts in each group, we first computed regression coefficients from the dataset of the other six years and applied these regression coefficients to the corrections. Note that MSLP is used to correct Vmax forecasts because the relationship between biases and deviation in potential intensities for MSLP exhibits a more linear trend than that for Vmax (Figs. 4e, 4f, 5e and 5f) and fits a linear regression. For this correction, physical environmental parameters SHEAR, CAPE and MSLP PI are calculated from predicted variables available at the initial time of the forecast to evaluate the benefits in realistic conditions. More specifically, (1) the center position used to calculate environmental conditions is changed from the RSMC Tokyo best track to the location of the MSLP in the JMA-GSM forecast, and (2) relative humidity, 
(a)

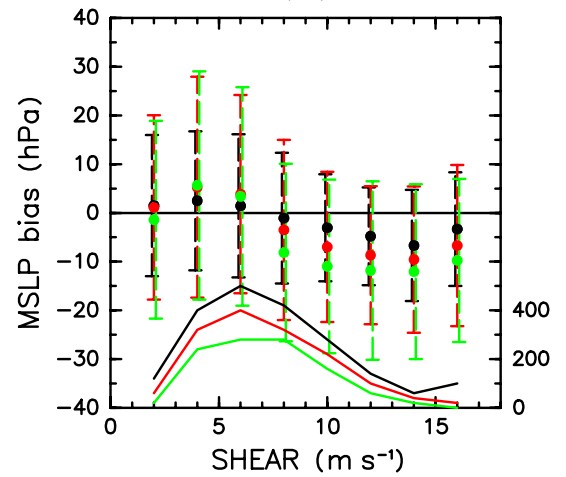

(d)

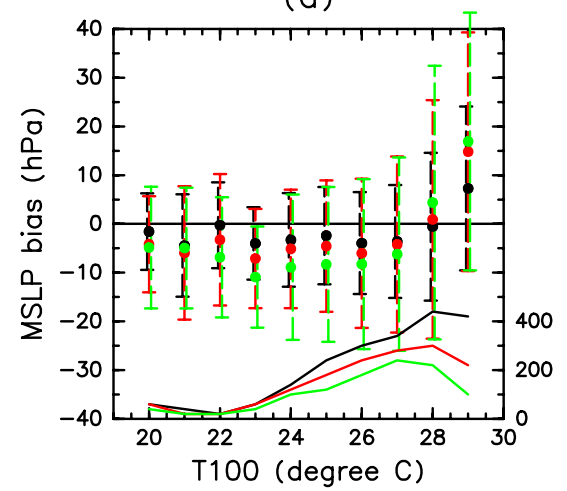

(b)

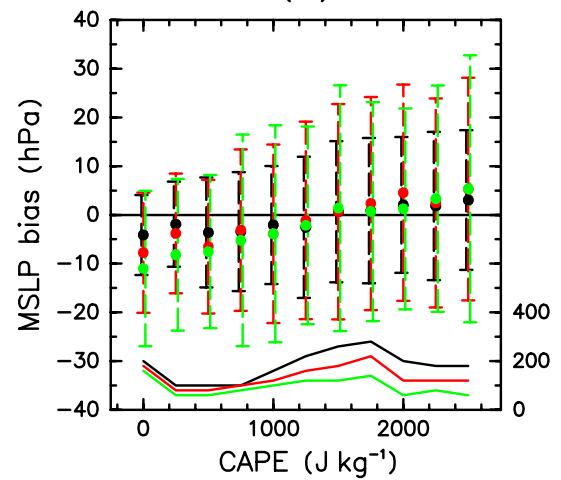

(e)

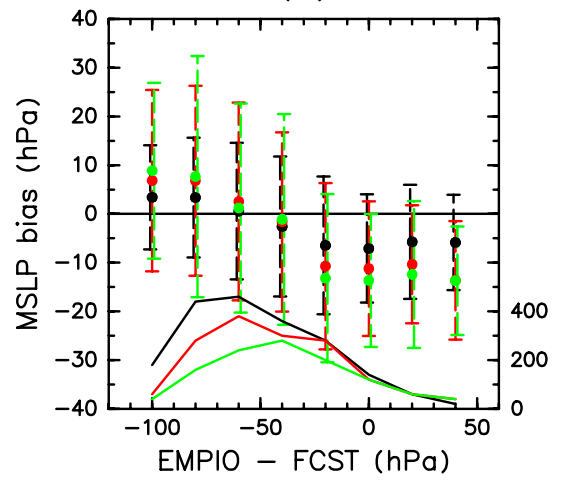

(c)

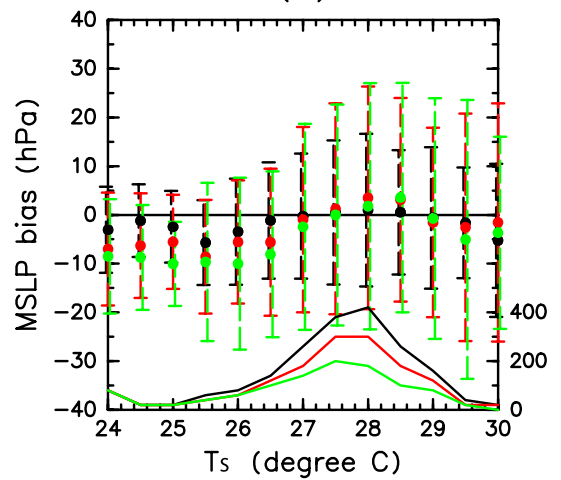

(f)

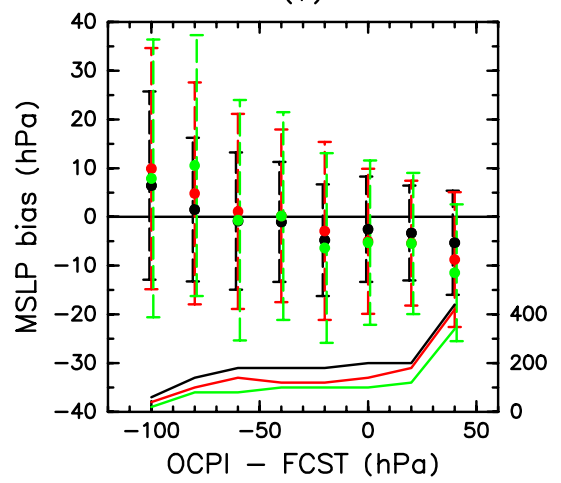

Fig. 4. Biases in the MSLP forecasts stratified according to physical environmental parameters: (a) magnitude of the vertical shear of horizontal wind, (b) CAPE, (c) $T_{s}$, (d) $T_{100}$, and (e) deviation in MSLP derived from E-MPIO relative to MSLP derived from RSMC Tokyo forecast. (f) Same as (e) but for MSLP derived from OC PI, respectively. Dots represent the mean biases in each category and the vertical bar centered at the dot represents the standard deviation of errors in forecasts published by RSMC Tokyo. Solid lines indicate the number of cases. Left vertical axis is for the mean bias and standard deviation, while the right axis corresponds to the number of cases. Black, red, and green indicate the results of FT $=24,48$, and $72 \mathrm{~h}$, respectively.

(a)

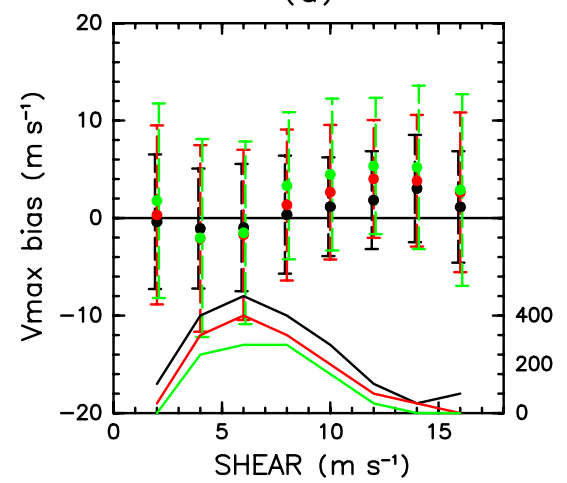

(d)

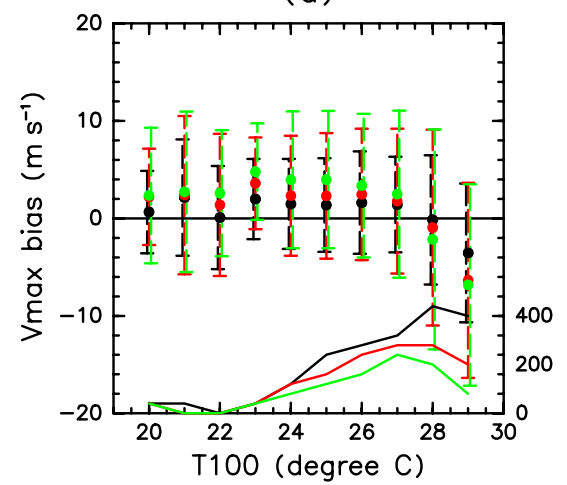

(b)

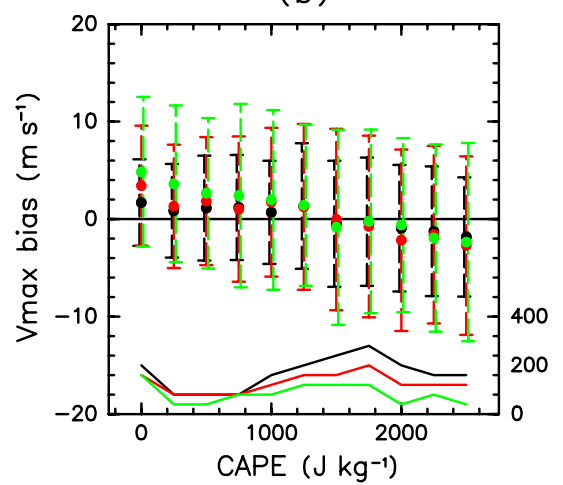

(e)

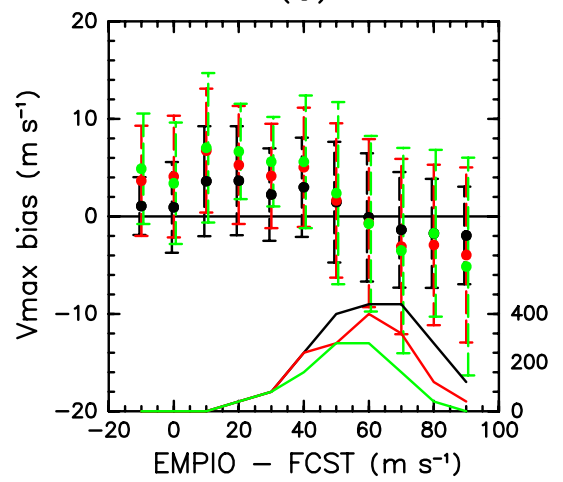

(c)

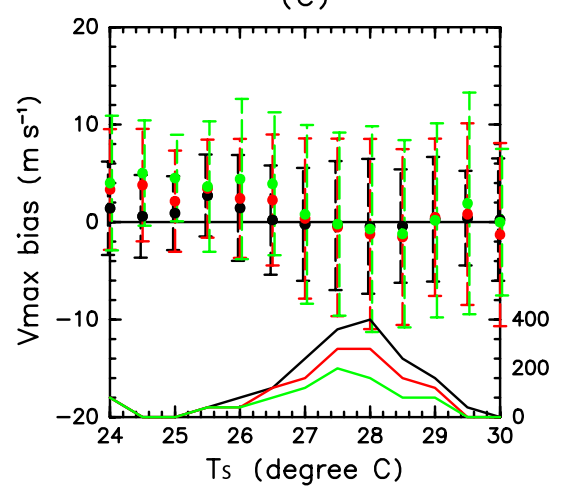

(f)

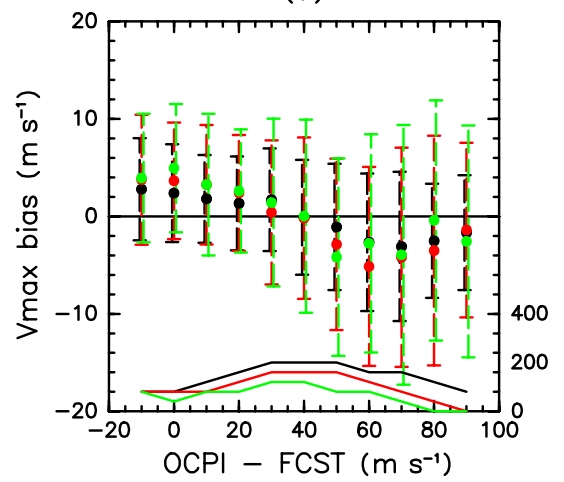

Fig. 5. Vmax biases according to physical environmental parameters, as defined for Fig. 4. Note that the horizontal axes represent potential intensities for Vmax in (e) and (f) rather than for MSLP. 
Table 1. Regression coefficients, RMSEs of $F$ and $F^{\prime}$ in Eq. (4), and the improvement rate $\left(F^{\prime}-F\right) / F$. Results are averaged over seven groups divided according to the year and standard deviation over seven samples for RMSE and improvement rate is indicated by a number after plus-minus sign.

\begin{tabular}{cccccccccr}
\hline Exp. & $\mathrm{FT}$ & $\alpha_{1}$ & $\alpha_{2}$ & $\alpha_{3}$ & $\alpha_{4}$ & $\beta$ & $\begin{array}{c}\text { RMSE } \\
(F)\end{array}$ & $\begin{array}{r}\text { RMSE } \\
\left(F^{\prime}\right)\end{array}$ & $\begin{array}{c}\text { Improvement rate } \\
(\%)\end{array}$ \\
\hline MSLP & 24 & -0.37 & 0.0030 & -0.010 & 0.20 & -183 & $13.5 \pm 0.9$ & $12.6 \pm 0.7$ & $6.4 \pm 3.4$ \\
(E-MPIO) & 48 & -1.09 & 0.0043 & -0.051 & 0.36 & -297 & $20.0 \pm 1.8$ & $18.0 \pm 1.7$ & $9.5 \pm 4.9$ \\
& 72 & -2.09 & 0.0050 & -0.057 & 0.43 & -358 & $22.1 \pm 1.9$ & $19.4 \pm 1.9$ & $12.1 \pm 5.5$ \\
\hline MSLP & 24 & -0.36 & 0.0029 & -0.041 & 0.21 & -163 & $13.5 \pm 0.9$ & $12.2 \pm 0.7$ & $9.4 \pm 3.4$ \\
(OC_PI) & 48 & -1.10 & 0.0042 & -0.081 & 0.38 & -291 & $19.9 \pm 1.8$ & $17.1 \pm 1.5$ & $14.1 \pm 3.1$ \\
& 72 & -2.03 & 0.0051 & -0.094 & 0.47 & -355 & $22.1 \pm 1.9$ & $18.4 \pm 1.8$ & $16.9 \pm 4.2$ \\
\hline MSLP & 24 & -0.05 & 0.0018 & -0.004 & 0.35 & -344 & $10.5 \pm 1.9$ & $8.3 \pm 2.0$ & $21.1 \pm 10.2$ \\
(E-MPIL) & 48 & -0.26 & 0.0032 & -0.002 & 0.54 & -531 & $16.3 \pm 2.2$ & $11.0 \pm 2.0$ & $32.0 \pm 11.4$ \\
& 72 & -0.47 & 0.0026 & -0.002 & 0.67 & -659 & $20.6 \pm 1.6$ & $13.3 \pm 2.1$ & $35.3 \pm 8.7$ \\
\hline Vmax & 24 & 0.12 & -0.0013 & 0.007 & -0.07 & 65 & $6.2 \pm 0.5$ & $5.9 \pm 0.5$ & $4.9 \pm 3.0$ \\
(E_MPIO) & 48 & 0.41 & -0.0018 & 0.025 & -0.13 & 105 & $8.6 \pm 1.0$ & $7.9 \pm 1.0$ & $7.7 \pm 3.2$ \\
& 72 & 0.84 & -0.0019 & 0.028 & -0.16 & 125 & $9.3 \pm 0.7$ & $8.3 \pm 0.8$ & $10.8 \pm 5.4$ \\
\hline Vmax & 24 & 0.12 & -0.0012 & 0.021 & -0.08 & 56 & $6.2 \pm 0.5$ & $5.7 \pm 0.5$ & $7.8 \pm 2.7$ \\
(OC_PI) & 48 & 0.41 & -0.0017 & 0.037 & -0.14 & 103 & $8.6 \pm 1.0$ & $7.5 \pm 1.0$ & $12.6 \pm 2.1$ \\
& 72 & 0.81 & -0.0021 & 0.041 & -0.17 & 126 & $9.3 \pm 0.7$ & $7.9 \pm 0.9$ & $15.7 \pm 6.3$ \\
\hline Vmax & 24 & 0.05 & -0.0009 & 0.004 & -0.20 & 191 & $6.4 \pm 1.2$ & $5.7 \pm 0.8$ & $10.9 \pm 7.2$ \\
(E_MPIL) & 48 & 0.20 & -0.0013 & 0.004 & -0.29 & 283 & $9.1 \pm 1.1$ & $7.0 \pm 0.4$ & $22.5 \pm 9.1$ \\
& 72 & 0.34 & -0.0005 & 0.002 & -0.36 & 351 & $11.2 \pm 1.8$ & $8.2 \pm 1.8$ & $26.5 \pm 9.0$ \\
\hline
\end{tabular}

temperature profile, CAPE, $T_{S}$, and $T_{100}$ at the JMA-GSM forecast center position 3 days prior to the verification time for $\mathrm{FT}=72 \mathrm{~h}$ are used.

Table 1 describes the optimized coefficients as well as the improvement rate (defined as percentage of reduction in RMSE) compared to the official forecasts of RSMC Tokyo. As expected, TC intensity forecasts over the open ocean are improved by $4.9-12.1 \%$ and $7.8-16.9 \%$ when E-MPIO and OC PI are used as a potential intensity, respectively. Improvement rates become larger with increasing FTs or by employing OC PI. The forecasts are also improved by $10.9-35.3 \%$ for a TC that made landfall or is around a coastal region. These improvements for both Vmax and MSLP at each FT are statistically significant, with at least the $99 \%$ confidence level, when applying a paired sample two-tailed $t$-test to seven groups of RMSEs. The total RMSE of corrected forecasts on each year was calculated using an OC_PI (open ocean) and E-MPI (coastal region and land) (Fig. 2). It exhibits the robust reduction of RMSE across all years. Comparing the corrected forecasts during 2008-2014 with operational forecasts until 2007, RMSEs of corrected forecasts were statistically smaller in terms of MSLP at FT $=72 \mathrm{~h}$ with the confidence level of $95 \%$, while any other difference did not reach the confidence level of $95 \%$.

\section{Discussion}

An interesting result is that the long-term trend of intensity forecast error shows an increasing behavior. A possible explanation is that a rapid change of TC intensity has occurred frequently over the last 10 years (Fig. 6). Such frequency of rapid intensification is positively correlated with RMSEs (Figs. 2b and c). The difficulty to predict rapid changes in TC intensity (Fig. 3) could yield the behavior of TC intensity error. This increase of rapid intensification events is also seen in the dataset published by the Joint Typhoon Warning Center (Wang et al. 2015). Nevertheless, the reason for the increase of rapid intensification events is still not clear. It could result from a climatological feature (Pun et al. 2013) or merely an artifact due to changes in the policy of constructing a best track dataset, Dvorak tables, and collecting observations.

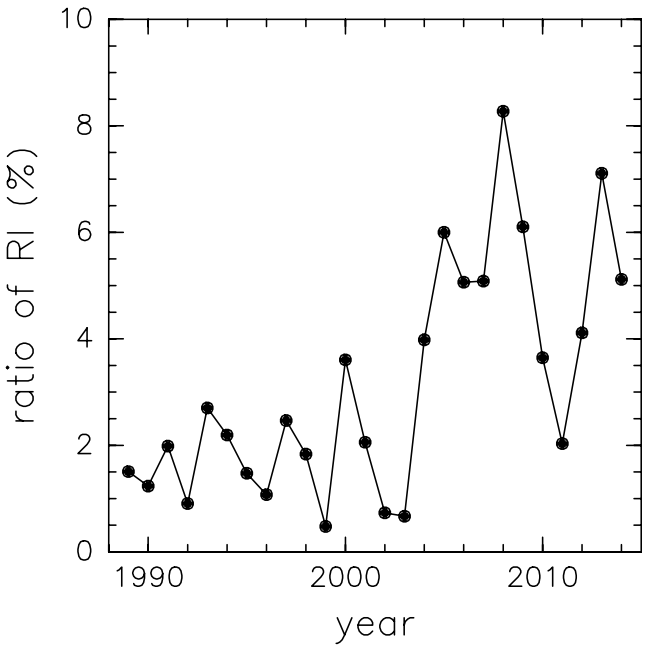

Fig. 6. Occurrence rate of rapid intensification in the RSMC Tokyo best track.

\section{Concluding remarks}

Errors in operational TC intensity forecasts by the RSMC Tokyo were analyzed based on their annual reports. This analysis reveals that (1) annual mean forecasts accuracy has not improved in over 26 years and (2) forecast errors tend to be larger in developing and decaying stages. Although official TC intensity forecasts rely on many components including numerical modeling, data assimilation schemes, observations, statistical correction, and forecaster's skill, we found that recent official forecasts contain biases with respect to the vertical wind shear, CAPE, UOT, and potential intensities. The proposed simple statistical correction shows the potential usefulness of taking these environmental parameters into account.

This statistical model can be refined by tuning the horizontal or temporal scale of represented environmental conditions, considering the TC translation speed (Lin et al. 2008) and the vertical wind shear at different elevations. Moreover, sophisticated oceanic data allow for further improvements in the forecast accuracy 
(Supplemental material B). Moreover, a 20-km mesh atmospheric model is currently used in JMA as a base model. The use of a high-resolution atmosphere-ocean coupled model can enhance accuracy as exemplified by Ito et al. (2015) for TC intensity forecasts around Japan.

Please remind that the present work merely addresses the status of TC intensity forecast system by the RSMC Tokyo to date. Coefficients should be adjusted again when these biases are recognized by the forecasters or when a base numerical model is changed. Therefore, it is also important to develop a stable statistical guidance, which is briefly discussed in supplemental material B. Moreover, the current result may depend on the choice of the best track dataset because the dataset is substantially different among operational centers. Nevertheless, the present work clarifies the nature of the RSMC Tokyo official TC intensity errors and the room for improvement by relating the forecast errors and environmental parameters, which can benefit the WNP region.

\section{Acknowledgment}

We thank Ms. Yui Miyazato, Mariko Nakada, Natsumi Yoshimura, and Mr. Narumichi Miyamoto and Shouta Kanda for digitizing a dataset and Drs. M. Sawada, H. Yamada, and M. Yamaguchi for valuable comments. This work is supported by MEXT KAKENHI Grant 25400461 and 16K05556. The figures were produced by GFD-DENNOU Library.

Edited by: H. Kamahori

\section{Supplement}

Supplementary materials include 2 figures and 2 tables.

\section{References}

Bhatia, K. T., and D. S. Nolan, 2013: Relating the skill of tropical cyclone intensity forecasts to the synoptic environment. Wea. Forecasting, 28, 961-980.

Bister, M., 2002: Low frequency variability of tropical cyclone potential intensity 1 . Interannual to interdecadal variability. $J$. Geophys. Res. Atmos., 107.

Bister, M., and K. A. Emanuel, 1998: Dissipative heating and hurricane intensity. Meteor. Atmos. Phys., 65, 233-240.

D'Asaro, E. A., and co-authors, 2011: Typhoon-ocean interaction in the western North Pacific: Part 1. Oceanography, 24, 2431.

Emanuel, K., C. DesAutels, C. Holloway, and R. Korty, 2004:
Environmental control of tropical cyclone intensity. $J$. Atmos. Sci., 61, 843-858.

Emanuel, K. A., 1986: An air-sea interaction theory for tropical cyclones. Part I: Steady-state maintenance. J. Atmos. Sci., 43, 585-605.

Ito, K., T. Kuroda, K. Saito, and A. Wada, 2015: Forecasting a large number of tropical cyclone intensities around Japan using a high-resolution atmosphere-ocean coupled model. Wea. Forecasting, 30, 793-808.

JMA: Outline of the operational numerical weather prediction at the Japan Meteorological Agency (Available online at http:// www.jma.go.jp/jma/jma-eng/jma-center/nwp/outline2013nwp/index.htm. on 1 May 2016.)

Koide, N., N. Kono, and K. Nagata, 2014: Country report, Japan Meteorological Agency. JMA/WMO Workshop on Effective Tropical Cyclone Warning in Southeast Asia, Tokyo, Japan.

Lin, I. I., and co-authors, 2013: An ocean coupling potential intensity index for tropical cyclones. Geophys. Res. Lett., 40 , 1878-1882.

Price, J., 2009: Metrics of hurricane-ocean interaction: verticallyintegrated or vertically-averaged ocean temperature? Ocean Science, 5, 351-368.

Pun, I. F., I. I. Lin, and M. H. Lo, 2013: Recent increase in high tropical cyclone heat potential area in the Western North Pacific Ocean. Geophys. Res. Lett., 40, 4680-4684.

Ueno, M., and M. Kunii, 2009: Some aspects of azimuthal wavenumber-one structure of typhoons represented in the JMA operational mesoscale analyses. J. Meteor. Soc. Japan, 87, 615-633.

Usui, N., S. Ishizaki, Y. Fujii, H. Tsujino, T. Yasuda, and M. Kamachi, 2006: Meteorological Research Institute multivariate ocean variational estimation (MOVE) system: Some early results. Adv. Space Res., 37, 806-822.

Usui, N., and co-authors, 2016: Four-dimensional variational ocean reanalysis: A 30-year high-resolution dataset in the western North Pacific (FORA-WNP30), J. Oceanogr. (submitted).

Wang, X., C. Wang, L. Zhang, and X. Wang, 2015: Multidecadal variability of tropical cyclone rapid intensification in the western North Pacific. J. Climate, 28, 3806-3820.

Wang, Y., and C.-C. Wu, 2004: Current understanding of tropical cyclone structure and intensity changes-a review. Meteor. Atmos. Phys., 87, 257-278.

Yu, H., P. Chen, Q. Li, and B. Tang, 2013: Current capability of operational numerical models in predicting tropical cyclone intensity in the western North Pacific. Wea. Forecasting, 28, 353-367.

Manuscript received 2 June 2016, accepted 12 August 2016 SOLA: https://www.jstage.jst.go.jp/browse/solal 